\title{
Poor adherence to anticoagulation guidelines in patients with non-valvular atrial fibrillation treated in a tertiary cardiology unit
}

\author{
Guan Sen Kew, ${ }^{1}$ Mabel Tan, ${ }^{2}$ Toon Wei Lim ${ }^{1,3}$
}

${ }^{1}$ National University Hospital, Singapore, Singapore ${ }^{2}$ Singapore General Hospital, Singapore, Singapore ${ }^{3}$ National University Heart Centre, Singapore, Singapore

\section{Correspondence to} Guan Sen Kew, National University Hospital, 1E Kent Ridge Road, NUHS Tower Block, Level 9, Singapore 119228, Singapore; kewguansen@gmail.com

Received 27 November 2014 Revised 8 March 2015 Accepted 26 March 2015

\section{CrossMark}

To cite: Kew GS, Tan M Lim TW. Heart Asia

2015;7:18-22.

doi:10.1136/heartasia-2014010600

\section{ABSTRACT}

Introduction Atrial fibrillation (AF) is a well-known cause of strokes and all major society guidelines recommend oral anticoagulants (OAC) such as vitamin $\mathrm{K}$ antagonists (VKA) for patients with concomitant risk factors to prevent them. However, compliance with these guidelines is historically poor. This study aims to evaluate the adherence to $\mathrm{CHADS}_{2}$ (congestive heart failure, hypertension, age $\geq 75$ years, diabetes mellitus and prior stroke or transient ischaemic attack) guidelines, explore reasons and evaluate outcomes for non-adherence in a tertiary cardiology unit.

Materials and methods A retrospective study of patients admitted into a tertiary cardiology unit from January to March 2010.

Results of 1826 unique cardiac patients screened, 163 $(8.9 \%)$ of them had non-valvular AF or atrial flutter. Their mean age was 69.8 years and $58.9 \%$ were men. Of the 54 patients on warfarin with documented international normalised ratio (INR), only 22 (40.7\%) of them had an INR within the therapeutic range (INR less than two in $22(40.7 \%)$ and greater than three in 10 $(18.5 \%))$. Of the 119 patients with $\mathrm{CHADS}_{2}$ greater than or equal to two, only $46(38.7 \%)$ were discharged with warfarin. Among the remaining $73(61.3 \%)$ patients, the most common reasons for not prescribing warfarin include history of bleeding $(n=24,32.9 \%)$, no reason documented $(n=17,23.3 \%)$ and patient preference $(n=12,19.2 \%)$. On follow-up, patients on warfarin were found to have better survival outcomes (mean 1522 \pm 41 days) as compared with (mean $1255 \pm 63$ days) in patients not on OAC ( $p$ value $=0.001$ ).

Conclusions Few patients who require anticoagulation receive it in accordance with the guidelines even in a tertiary cardiology unit. There are many impediments to the effective use of VKA for stroke prevention among patients with AF.

\section{BACKGROUND}

Atrial fibrillation (AF) is the most common chronic cardiac arrhythmia encountered in clinical practice. The overall prevalence of $\mathrm{AF}$ is $5.5 \%$ in adults aged $>55$ years, and this increases significantly with age, rising from $0.7 \%$ in those aged between 55 and 59 years to $17.8 \%$ in those aged $>85$ years. ${ }^{1} \mathrm{AF}$ is common and a strong risk factor for stroke, resulting in significant morbidity and mortality.

The effectiveness of oral anticoagulation (OAC) therapy such as vitamin $\mathrm{K}$ antagonists (VKA; eg, warfarin) in preventing a thromboembolic event in patients with AF has been well studied in large randomised trials. ${ }^{2-4}$ Currently, all major published guidelines recommend $\mathrm{OAC}$ for patients at moderate or high risk of stroke, aiming for a therapeutic international normalised ratio (INR) of two to three if the patient is treated with a VKA. Antiplatelets or no antithrombotic therapy at all is indicated in patients at moderate or low risk..$^{5-7}$

Despite the extent of data and the presence of clear guidelines for more than a decade, the realworld benefit of warfarin may be much lower than that demonstrated in clinical trials. Adherence to anticoagulation guidelines has been historically poor in multiple studies from various countries. ${ }^{8} 9$ A recent systemic review demonstrated that patients in close to $90 \%$ of the studies were under-treated with OAC therapy (treatment level $<70 \%$ of highrisk patients). ${ }^{10}$ The reasons for such low rates of compliance are often obscure. Till date, there has been no study in Singapore looking into our adherence to $\mathrm{CHADS}_{2}$ (congestive heart failure, hypertension, age $\geq 75$ years, diabetes mellitus and prior stroke or transient ischaemic attack) guidelines except for a small-scale study done in a geriatric unit in 2002 which found that among these patients, very few were treated with anticoagulation. ${ }^{11}$ Since then, there has been no follow-up study on anticoagulation use in patients with $\mathrm{AF}$ and it would be timely to again examine our adherence to anticoagulation guidelines.

The $\mathrm{CHADS}_{2}$ score is one of the three stroke risk stratification schemes (besides stroke prevention in AF and Framingham score) shown to have greater predictive accuracy for stroke than chance. $^{12}$

This study aims to evaluate the adherence to contemporary anticoagulation guidelines among patients admitted to a tertiary cardiac unit and to explore reasons for withholding VKA in these patients. For the purposes of identifying high-risk patents in this study, the $\mathrm{CHADS}_{2}$ score was used, instead of the updated $\mathrm{CHA}_{2} \mathrm{DS}_{2}$-VASc score, because the latter scoring system had not yet been incorporated into practice guidelines in our study period in the first quarter of $2010 .^{13}$

\section{METHODOLOGY}

We reviewed consecutive patients admitted into a tertiary cardiology unit from January to March 2010 to identify patients with a history of or presenting with non-valvular AF or atrial flutter through an electronic medical records system. Readmission cases were excluded from the study and only index admissions from the 3-month period were considered. Patients with atrial flutter were included, since guidelines regarding anticoagulation follow the same recommendations as for 
$\mathrm{AF}^{14}$ The rationale for selecting this period in time was to allow a maximal amount of time since the publication of the previous $\mathrm{AF}$ anticoagulation guidelines in $2006^{6}$ and just prior to the switch to the recommendation to use the CHADS-VASc score in the European Society of Cardiology guidelines on 29 August 2010 to ensure that physicians had an opportunity to familiarise themselves with the contemporary guidelines.

For each patient, the electronic medical record was reviewed, including the discharge summary, laboratory and radiology results, prescription records and outpatient follow-up records. In selected cases, physical progress notes were also reviewed to clarify any areas of doubt. The data captured included demographic data, history of AF (duration, type, INR on admission) and how it was managed on admission and at discharge, other medical comorbidities, $\mathrm{CHADS}_{2}$ score, contraindications to $\mathrm{OAC}$ and other reasons for withholding OAC therapy (if any) were also evaluated. Patients were also reviewed after discharge to look for thromboembolic stroke, systemic embolisation, allcause mortality or other clinical events.

For patients with a $\mathrm{CHADS}_{2}$ score of greater than or equal to two and yet not on any OAC, their old discharge summaries and ward progress notes were traced to determine whether they were ever on warfarin and the events that led to discontinuation or withholding of warfarin.

\section{Statistical analysis}

Continuous variables are expressed as mean \pm SD unless they have a non-normal distribution, in which case they are expressed as median (IQR). Analysis was performed on SPSS V.14 (SPSS, Chicago, Illinois, USA). Continuous-dependent variables at a single time point were analysed using the Student $t$ test. Predictors of being prescribed warfarin at discharge were analysed using binary logistical regression or $\chi^{2}$ test where appropriate. Survival analysis was performed using KaplanMeier and Cox regression methods. A p value of $<0.05$ was considered significant for all analyses.

\section{RESULTS}

\section{Basic demographics}

Of a total of 1826 cardiac patients screened, there were 163 (8.9\%) non-valvular $\mathrm{AF}$ or atrial flutter patients (mean age $69.8 \pm 13.3$ years, $59 \%$ men). The ethnic distribution was 123 (76\%) Chinese, 26 (16\%) Malays, seven (4\%) Indians, two (1\%) Eurasians and five $(3 \%)$ others. Among patients with non-valvular $\mathrm{AF}(\mathrm{n}=151)$, there were $24(16 \%)$ patients with newly diagnosed AF, $71(47 \%)$ patients with paroxysmal AF, five (3\%) patients with persistent $\mathrm{AF}$ and 51 (34\%) patients with long-standing persistent AF. There were 12 patients with atrial flutter only, of which three of them were newly diagnosed at the index admission.

The mean $\mathrm{CHADS}_{2}$ score was $2.41 \pm 1.49$, with majority of the patients having a $\mathrm{CHADS}_{2}$ score of two $(n=44,27 \%)$ or three $(n=43,26 \%)$, indicating an elevated risk of stroke.

\section{Use of warfarin in patients}

Of the 119 patients with $\mathrm{CHADS}_{2}$ score greater than or equal to two, only 42 were treated with warfarin at admission and this frequency was similar to that among patients with a $\mathrm{CHADS}_{2}$ score of less than two (35\% vs $30 \%$ respectively, $p=0.491)$. At discharge, the frequency of being prescribed warfarin between these two groups was also similar (39\% vs 36\%, $\mathrm{p}=0.789$ ).

There were 77 patients with $\mathrm{CHADS}_{2}$ greater than or equal to two, but were not on any warfarin on admission. Of these, there were 50 known cases, while the other 27 were newly diagnosed on their index admission. In the former group, only eight
Table 1 Baseline characteristics of patients discharged with warfarin and not on warfarin

\begin{tabular}{llll}
\hline & $\begin{array}{l}\text { Warfarin } \\
(\mathbf{n}=62)\end{array}$ & $\begin{array}{l}\text { Not on warfarin } \\
(\mathbf{n}=101)\end{array}$ & $\mathbf{p}$ Value \\
\hline Mean age (years) & $68.4 \pm 10.1$ & $72.1 \pm 15.0$ & 0.09 \\
Female & $30(48 \%)$ & $44(44 \%)$ & 0.19 \\
Congestive heart failure & $27(44 \%)$ & $53(53 \%)$ & 0.23 \\
Hypertension & $47(76 \%)$ & $70(69 \%)$ & 0.97 \\
Age $\geq 75$ years & $19(31 \%)$ & $48(48 \%)$ & 0.01 \\
Diabetes & $25(40 \%)$ & $38(38 \%)$ & 0.97 \\
Stroke/TIA & $16(25.8 \%)$ & $15(15 \%)$ & 0.10 \\
CHADS $_{2}$ score & $2.47 \pm 1.46$ & $2.38 \pm 1.52$ & 0.67 \\
\hline
\end{tabular}

CHADS2, congestive heart failure, hypertension, age $\geq 75$ years, diabetes mellitus and prior stroke or transient ischaemic attack; TIA, transient ischaemic attack.

of 50 patients $(16 \%)$ were offered warfarin on discharge, whereas in the latter group, 15 of 27 (56\%) patients were offered warfarin. Hence, newly diagnosed patients with AF were more likely to be offered warfarin compared with known cases (OR 6.56, $\mathrm{p}=0.001$ ). Baseline characteristics comparing patients discharged with warfarin and not on warfarin are tabulated in table 1 .

\section{Reasons for not anticoagulating}

Among patients with $\mathrm{CHADS}_{2}$ greater than or equal to two, the most common reason for not prescribing warfarin (table 2) was a history of bleeding, followed by no reason documented and patient preference. It was however important to note that most bleeding episodes $(n=16,67 \%)$ were remote episodes in the past (bleeding more than a year ago) and there was no active bleeding at the point of admission in any of these patients. Patients who objected to warfarin use tend to be older (mean age $77.5 \pm 10.7$ years), compared with patients who were on warfarin (mean age $68.4 \pm 10.1$ ). Other reasons for withholding warfarin therapy are tabulated in table 3 .

\section{INR on admission}

Among patients who were already on warfarin prior to admission $(n=55)$, their admission INR ranged from 0.86 to $>7.50$. Only $22(40 \%)$ patients had an INR within the therapeutic range of two to three. Another $22(40 \%)$ had an INR less than two and $10(18 \%)$ had an INR greater than three. The INR of one patient could not be found.

Table 2 Reasons for not anticoagulating

\begin{tabular}{lc}
\hline Reason & No. of patients $(\mathbf{n}=73)$ \\
\hline Bleeding & $24(32.9 \%)$ \\
No reason documented & $17(23.3 \%)$ \\
Refused warfarin & $14(19.2 \%)$ \\
Unexplained anaemia & $12(16.4 \%)$ \\
Risk of falls & $10(13.7 \%)$ \\
Non-compliance & $6(8.2 \%)$ \\
Poor life expectancy & $5(6.8 \%)$ \\
Dementia/cognitive impairment & $5(6.8 \%)$ \\
Poor social support & $2(2.7 \%)$ \\
\hline Numbers do not add up to $73(100 \%)$ as there may be more than one reasons for \\
withholding warfarin.
\end{tabular}


Table 3 Predictors of being discharged on warfarin

\begin{tabular}{|c|c|c|c|}
\hline & OR & $\mathrm{Cl}$ & p Value \\
\hline \multicolumn{4}{|l|}{ Univariate analysis } \\
\hline Congestive heart failure & 0.70 & 0.37 to 1.32 & 0.269 \\
\hline Hypertension & 1.34 & 0.68 to 2.85 & 0.372 \\
\hline Age $\geq 75$ years & 0.49 & 0.25 to 0.95 & 0.035 \\
\hline Diabetes & 1.12 & 0.59 to 2.14 & 0.731 \\
\hline Stroke/TIA & 2.17 & 0.99 to 4.74 & 0.053 \\
\hline Vascular disease & 0.96 & 0.51 to 1.82 & 0.900 \\
\hline Female & 1.27 & 0.67 to 2.39 & 0.469 \\
\hline Warfarin on admission & 46.0 & 17.4 to 121 & $<0.001$ \\
\hline Bleeding & 0.25 & 0.09 to 0.70 & 0.008 \\
\hline CHADS $\geq 2$ & 1.10 & 0.54 to 2.26 & 0.789 \\
\hline Paroxysmal AF & 0.81 & 0.43 to 1.54 & 0.514 \\
\hline Admission diagnosis of AF & 1.04 & 0.51 to 2.11 & 0.924 \\
\hline \multicolumn{4}{|l|}{ Multivariate analysis } \\
\hline Warfarin on admission & 47.5 & 17.2 to 130.7 & $<0.001$ \\
\hline Bleeding & 0.23 & 0.55 to 0.93 & 0.039 \\
\hline
\end{tabular}

\section{Predictors of VKA prescription}

Predictors of patients being discharged on warfarin were analysed using logistical regression (table 4). Treatment with warfarin prior to admission predicted being discharged with it (OR 46.0, $\mathrm{p}<0.001$ ), while a history of bleeding made it less likely (OR $0.25, p=0.008$ ). Despite age $\geq 75$ years being an important risk factor for stroke, patients in this category were less likely to be on OAC therapy (OR 0.49, p=0.035). There was a nearly significant trend of having a previous stroke being predictive of warfarin use.

Using multivariate analysis of individual predictors with $\mathrm{p}$ value of $<0.100$, only warfarin on admission (OR 47.5, 95\% CI 17.2 to $130.7, \mathrm{p}<0.001$ ) and bleeding (OR $0.23,95 \%$ CI 0.55 to $0.93, p=0.039$ ) remained statistically significant.

\section{Follow-up period}

Patients were followed up for up to 4.5 years following their index admission. There were 37 (23\%) patients who died during this period. Major causes of death include sepsis $(n=10$, 27\%), cardiac disease (acute myocardial infarction or congestive heart failure; $n=7,19 \%)$ and stroke $(n=5,14 \%)$. It was also found that $19(12 \%)$ patients had an ischaemic stroke during the same period, of which five of them died during the same

Table 4 Cox regression of factors affecting mortality and stroke

\begin{tabular}{lllll}
\hline & OR & Cl (95\%) & $\begin{array}{l}\text { Univariate } \\
\text { analysis, } \\
\text { p value }\end{array}$ & $\begin{array}{l}\text { Multivariate } \\
\text { analysis, } \\
\text { p value }\end{array}$ \\
\hline Warfarin & 0.32 & 0.16 to 0.65 & 0.002 & 0.008 \\
Congestive heart failure & 2.24 & 1.25 to 4.03 & 0.007 & 0.024 \\
Hypertension & 1.00 & 0.54 to 1.87 & 0.990 & \\
Age $>75$ years & 1.75 & 0.9984 to 3.09 & 0.053 & \\
Diabetes & 1.14 & 0.64 to 2.03 & 0.648 & \\
Vascular disease & 1.15 & 0.65 to 2.03 & 0.635 & \\
Female gender & 0.76 & 0.43 to 1.36 & 0.363 & \\
History of bleeding & 1.54 & 0.78 to 3.01 & 0.213 & \\
\hline
\end{tabular}

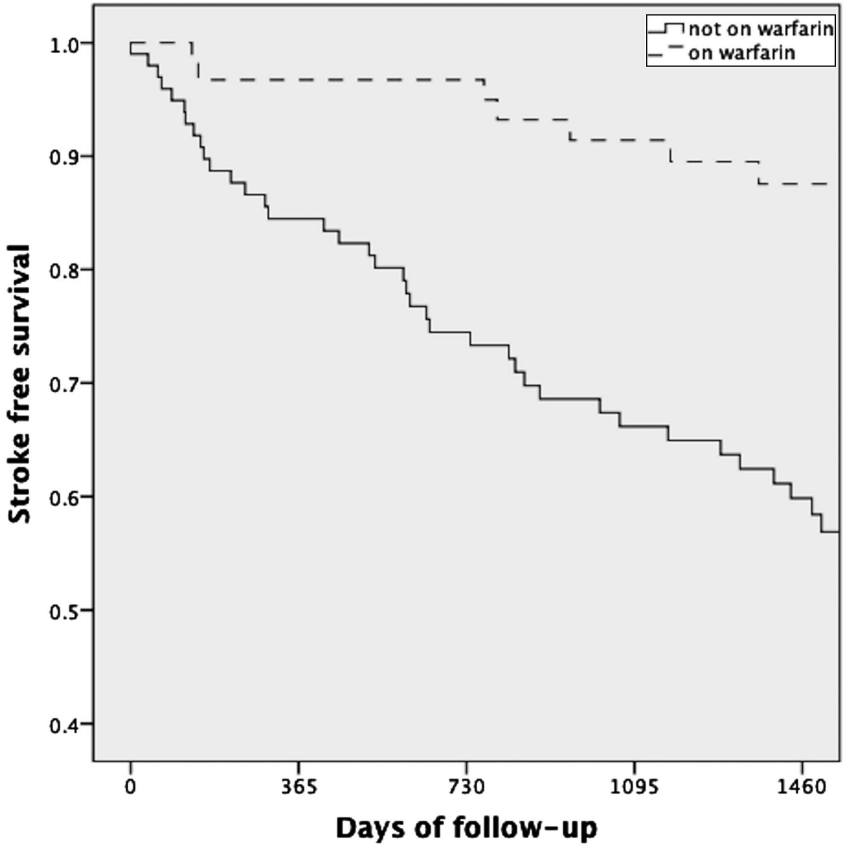

Figure 1 Kaplan-Meier curves of survival free of ischaemic stroke according to warfarin use.

admission. Among these patients with stroke, majority of them $(n=15,79 \%)$ were not on warfarin.

Kaplan-Meier survival plot with an endpoint of all-cause mortality or stroke is shown in figure 1 . When stratified according to whether patients were discharged with warfarin, the survival rates differed significantly between the different groups $(p=0.001)$. The mean stroke-free survival time for patients discharged with warfarin was significantly longer at $1522 \pm 41$ days than at $1255 \pm 63$ days in patients not on warfarin $(p=0.001)$. At 2 years after discharge, stroke-free survival was $95 \pm 3 \%$ for patients on warfarin versus $73 \pm 5 \%$ for patients discharged not on warfarin. Both time to all-cause mortality and strokes were also significantly longer in patients on warfarin.

Using Cox regression analysis, it was found that there was a significant association between OAC and congestive heart failure with survival. This is even so after taking into account of other possible confounders (age, gender, hypertension, diabetes mellitus, cardiovascular disease, history of bleeding). Patients who are on warfarin are less likely to have an event (OR 0.32, $\mathrm{p}=0.002$ ), while those with heart failure are more likely to have an event (OR 2.24, $\mathrm{p}=0.007$ ). Results of the Cox regression analysis are as shown in table 4 .

\section{DISCUSSION}

The main findings of this study are that many patients with nonvalvular AF, including the majority of those who are at high risk of a thromboembolic stroke were not treated with warfarin even among patients treated in a tertiary cardiac unit. The reasons for this poor adherence to well-known treatment guidelines are manifold, with chiefly a history of bleeding, patient preference, unexplained anaemia and risk of falls. In a substantial proportion of patients, the reason for the clinical decision not to initiate warfarin was not apparent from our retrospective study. The main predictors that affected whether a patient was discharged home on warfarin were whether they had already been started on warfarin and whether they had a previous bleeding event, rather than wellvalidated clinical predictors of stroke risk. Furthermore, patients 
who were not treated with warfarin on discharge had markedly worse stroke-free survival on long-term follow up.

\section{Low rates of warfarinisation for stroke prevention in AF}

The risk of thromboembolic stroke in patients with AF is not homogeneous, and it has long been recognised that antithrombotic therapy needs to be tailored to each patient's individual risk profile. This is reflected in the practice guidelines from all the major professional societies which recommend using risk stratification to guide therapy. ${ }^{13}{ }^{15}$ Central to these recommendations is that high-risk patients should be treated with anticoagulation to reduce their risk of thromboembolic events. Yet, we found a low rate of patients being treated with warfarin for non-valvular AF despite their risk profile. In this cohort, whether you had a $\mathrm{CHADS}_{2}$ score of zero to one or two or more, only $36 \%$ and $39 \%$ of patients were discharged home on warfarin, respectively, with many patients being treated with antiplatelet agents. This is also consistent with the AF in Turkey: Epidemiology Registry study, which included 1745 patients with non-valvular AF, and it was found that only $42 \%$ of the high-risk patients were anticoagulated. ${ }^{16}$ However, in our study, of particular concern, there were six patients with $\mathrm{CHADS}_{2}$ score of two or more and were not on any form of antithrombotic therapy.

Patients often find warfarin therapy inconvenient due to the periodic blood tests required for dose titration. This is compounded in the elderly who may have mobility or access difficulties which may hamper their compliance with visits to the clinic for blood tests and follow-up. More may have to be done to educate our patients in accepting its use as patient refusal was the third most common reason for warfarin underuse. Patients who objected to warfarin use in this study tend to be older (mean age $77.5 \pm 10.7$ years), and it underlines the importance of improving acceptance among elderly patients.

On the other hand, physicians were most worried about bleeding, and the fear of causing a severe haemorrhage often overrides the desire to actively prevent a stroke. ${ }^{17} 18$ This was reflected in our study whereby patients were not started on warfarin because of concerns about bleeding episodes in the past, anaemia and the risk of falls. These made up $63 \%$ of all patients who were not anticoagulated, despite their high risk. Of note, most of these bleeding episodes were in the remote past but were still cited as reasons not to commence warfarin. Furthermore, nearly $20 \%$ were not commenced for reasons that were not apparent in the patient's records. This failure of documentation in the patient's medical records was a worrying find, as it might either mean that physicians failed to consider anticoagulation therapy or that they failed to document the reason. In both cases, it demonstrates a failure to follow well-defined evidence-based clinical practice and shows there is much scope for improvement.

Studies have shown that OAC, compared with antiplatelets only, confer significant additional benefit to high-risk patients, but only minimal benefit to low-risk patients. ${ }^{19-22}$ Despite such evidence, warfarin prescription rate in our study was similar in the two groups. It is possible that these patients were on warfarin for other indications given their multiple comorbidities, but this finding is in keeping with previous studies that showed that despite varying levels of stroke risk, patients with $\mathrm{AF}$ receive warfarin therapy at much the same frequency. ${ }^{23}$ The reasons for this deviation from evidence-based practice are uncertain and may be due to physicians perceiving higher than usual risk in these patients. More systematic study of this is needed.

\section{Suboptimal warfarinisation was common}

Among the 55 patients in our study who were anticoagulated prior to admission, 32 (58.1\%) of them had an INR outside the therapeutic range. Our study cohort consisted of cardiology inpatients who were acutely unwell and required hospitalisation and hence may not be representative of the overall AF population. However, the low frequency of INR readings in range is also a consistent finding in previous studies. Even in large clinical trials where warfarin was administered in specialised anticoagulation clinics, time within therapeutic range was typically in the $50 \%-60 \%$ range for patients randomised to VKAs. ${ }^{24} 25$

\section{Association of warfarin use and better survival outcomes}

The low-rate anticoagulation therapy coupled with poor INR control found in our study was all the more concerning because we found that after a 5-year follow-up, 15 of 19 patients who had an ischaemic stroke were not on anticoagulation. Most of them were in the high-risk group $(n=18,94.7 \%)$, and had a $\mathrm{CHADS}_{2}$ score of two or more. While it is impossible to determine whether these events could have been prevented with the appropriate use of $\mathrm{OAC}$ due to the retrospective nature of this study, it nonetheless reasonable to conclude that not being anticoagulated may have contributed to a higher risk of strokes.

Furthermore, with Kaplan-Meier survival analysis (figure 1), patients discharged with warfarin had better survival outcomes, and this is independent of other factors, even when age is considered. This could possibly be due to the fact that warfarin reduces the risk of ischaemic stroke and cardiovascular events, which are common causes of death in our population. Although warfarin is known to increase the risk of bleeding, in carefully selected patients, their mortality benefits would far outweigh the bleeding risks. This further emphasises the need for appropriate anticoagulation for patients with high stroke score.

\section{CLINICAL SIGNIFICANCE}

Despite numerous studies showing the benefit of OAC and the importance of proper risk stratification, ${ }^{12}$ the benefit of these studies cannot be translated into real benefit in patients if adherence to guidelines remains poor. Our study is one of the many worldwide, which makes an important observation, that the real-world application of practice guidelines for stroke prevention in patients with AF remains poor. In the present study, we found that this was true even among patients admitted under the cardiology service of a tertiary teaching hospital. This perhaps highlights that knowledge and understanding of the guidelines among physicians may not always translate into the application of these guidelines in clinical practice. It also underlines how little progress has been made with this issue in the past 10 years in Singapore, despite the great improvements in healthcare during this time. This suggests that current models of physician centric care are inadequate and that new approaches are needed.

It may be argued that the advent of novel OAC such as direct thrombin inhibitors and direct factor $\mathrm{Xa}$ inhibitors may make many of these issues with warfarin a thing of the past. ${ }^{26}$ These new drugs have a predictable and stable dose-related anticoagulant effect, and there is no need for regular laboratory monitoring of anticoagulation intensity. In the long run, clinical practice may gradually move away from VKA, towards these more userfriendly novel OAC. Nevertheless, VKAs remain the standard of treatment in AF stroke prevention in many countries due to the cost of these newer agents and will continue to be so while there is a paucity of evidence for the use of these novel agents 
in patients who have valvular-related AF. Hence, our immediate priority should still be to optimise our use of VKAs. The barriers faced are manifold and will require an integrated care approach that involves physician education, patient education, optimising a patient's experience with anticoagulation by making their clinical encounters more efficient through the use of specialised anticoagulation services and it may also be helpful to use decision support tools such as software algorithms. ${ }^{27}$

\section{CONCLUSIONS}

Few patients with AF who should receive anticoagulation for stroke prevention in accordance with guidelines are discharged home on warfarin, even among patients admitted to a tertiary cardiology unit. The consequences of not anticoagulating highrisk patients are dire, with poorer survival outcomes and increased risk of stroke. There are many impediments to the effective use of VKA for stroke prevention among patients with $\mathrm{AF}$ and these continue to persist over the last decade in Singapore.

Contributors GSK: Responsible for collecting and analysis of data, design of the research and drafting the manuscript. MT: Helped in collecting and analysis of data, editing of the manuscript. TWL: Responsible for design of the research, analysis of data and editing the final manuscript.

Competing interests None declared.

Provenance and peer review Not commissioned; externally peer reviewed.

\section{REFERENCES}

1 Heeringa J, van der Kuip DA, Hofman A, et al. Prevalence, incidence and lifetimerisk of atrial fibrillation: the Rotterdam study. Eur Heart J 2006; 27:949-53.

2 The Boston Area Anticoagulation Trial for Atrial Fibrillation Investigators. The effect of low-dose warfarin on the risk of stroke in patients with nonrheumatic atrial fibrillation. N Engl J Med 1990;323:1505-11.

3 [No authors listed]. Warfarin versus aspirin for prevention of thromboembolism in atrial fibrillation: Stroke Prevention in Atrial Fibrillation II Study. Lancet 1994:343:687-91.

4 Connolly SJ, Laupacis A, Gent M, et al. Canadian Atrial Fibrillation Anticoagulation (CAFA) Study. J Am Coll Cardiol 1991;18:349-55.

5 Singer DE, Albers GW, Dalen JE, et al. Antithrombotic therapy in atrial fibrillation: the Seventh ACCP Conference on Antithrombotic and Thrombolytic Therapy. Chest 2004;126(3 Suppl):429S-56S.

6 Fuster V, Rydén LE, Cannom DS, et al. ACC/AHA/ESC 2006 guidelines for the management of patients with atrial fibrillation. Circulation 2006;114:e257-354.

7 National Institute for Health and Clinical Excellence. NICE Clinical Guideline 36-Atrial Fibrillation: The Management of Atrial Fibrillation. London: National Institute for Health and Clinical Excellence, 2006.
8 Dores $\mathrm{H}$, Cardiga R, Ferreira $\mathrm{R}$, et al. Atrial fibrillation and thromboembolic risk: what is the extent of adherence to guidelines in clinical practice? Rev Port Cardiol 2011;30:171-80.

9 Wilke T, Groth A, Mueller S, et al. Oral anticoagulation use by patients with atrial fibrillation in Germany. Adherence to guidelines, causes of anticoagulation under-use and its clinical outcomes, based on claims-data of 183,448 patients. Thromb Haemost 2012;107:1053-65.

10 Ogilvie IM, Newton N, Welner SA, et al. Underuse of oral anticoagulants in atrial fibrillation: a systematic review. Am J Med 2010;123:638-645.e4.

11 Lew SJW, Lim JKH. Stroke prevention in elderly patients with atrial fibrillation. Singapore Med J 2002;43:198-201.

12 Baruch L, Gage BF, Horrow J, et al. Can patients at elevated risk of stroke treated with anticoagulants be further risk stratified? Stroke 2007;38:2459-63.

13 Camm AJ, Kirchhof P, Lip GY, et al. Guidelines for the management of atrial fibrillation: the Task Force for the Management of Atrial Fibrillation of the European Society of Cardiology (ESC). Eur Heart J 2010;31:2369-429.

14 Singer DE, Albers GW, Dalen JE, et al. Antithrombotic therapy in atrial fibrillation: American College of Chest Physicians Evidence-Based Clinical Practice Guidelines (8th edition). Chest 2008; 133:546S.

15 Anderson JL, Halperin JL, Albert NM, et al. Management of patients with atrial fibrillation (Compilation of 2006 ACCF/AHA/ESC and 2011 ACCF/AHA/HRS Recommendations). Circulation 2013;127:1916-26.

16 Ertas F, Eren NK, Kaya H, et al, AFTER Investigators. The atrial fibrillation in Turkey: Epidemiologic Registry (AFTER). Cardiol J 2013;20:447-52.

17 Rosenman $M B$, Baker $L$, Jing $Y$, et al. Why is warfarin underused for stroke prevention in atrial fibrillation? A detailed review of electronic medical records. Curr Med Res Opin 2012;28:1407-14.

18 Lotze U, Liebetrau J, Malsch I, et al. Medical treatment of patients with atrial fibrillation aged over 80 years in daily clinical practice: influence of age and CHADS (2) score. Arch Gerontol Geriatr 2010;50:36-41.

19 Van Walraven C, Hart RG, Singer DE, et al. Oral anticoagulants vs aspirin in nonvalvular atrial fibrillation: an individual patient meta-analysis. JAMA 2002;288:2441-8.

20 Hart RG, Benavente O, McBride R, et al. Antithrombotic therapy to prevent stroke in patients with atrial fibrillation: a meta-analysis. Ann Intern Med 1999;131:492-501.

21 Ezekowitz MD, Levine JA. Preventing stroke in patients with atrial fibrillation. JAMA 1999:281:1830-5.

22 Segal JB, McNamara RL, Miller MR, et al. Anticoagulants or antiplatelet therapy for non-rheumatic atrial fibrillation and flutter. Cochrane Database Syst Rev 2001;1: CD001938.

23 Zimetbaum PJ, Thosani A, Yu HT, et al. Are atrial fibrillation patients receiving warfarin in accordance with stroke risk? Am J Med 2010;123:446-53.

24 Connolly SJ, Ezekowitz MD, Yusuf $S$, et al. Dabigatran versus warfarin in patients with atrial fibrillation. N Engl J Med 2009:361:1139-51.

25 Patel MR, Mahaffey KW, Garg J, et al. Rivaroxaban versus warfarin in nonvalvular atrial fibrillation. N Engl J Med 2011;365:883-91.

26 Potpara TS, Polovina MM, Licina MM, et al. Novel oral anticoagulants for stroke prevention in atrial fibrillation: focus on apixaban. Adv Ther 2012;29:491-507.

27 Hendriks JM, de Wit R, Crijns HJ, et al. Nurse-led care vs. usual care for patients with atrial fibrillation: results of a randomized trial of integrated chronic care vs. routine clinical care in ambulatory patients with atrial fibrillation. Eur Heart $J$ 2012;33:2692-9. 